http://jmscr.igmpublication.org/home/ ISSN (e)-2347-176x ISSN (p) 2455-0450 crossref DOI: https://dx.doi.org/10.18535/jmscr/v7i10.141

\title{
Knowledge and skill based training on neonatal resuscitation protocol to MBBS interns posted in neonatal ward - A before and after study
}

\author{
Authors \\ Dr Himanshu Sekhar Sahoo ${ }^{1}$, Dr Sasmita Devi Agrawal ${ }^{2 *}$, Dr Lisa Sarangi ${ }^{3}$, \\ Dr Gautam Tripathy ${ }^{4}$, Dr Sidhant Swarup ${ }^{5}$, Dr Smruti Dash Mohapatra ${ }^{6}$ \\ *Corresponding Author \\ Dr Sasmita Devi Agrawal
}

\begin{abstract}
Background: Effective resuscitation at birth can prevent a large proportion of neonatal deaths. Competency in cardio-pulmonary resuscitation is a critical factor in determining successful outcomes in neonate. Use of simulation-based learning methodologies enhances performance in both simulated resuscitations and real-life clinical situations. Retention of skill is more important for the MBBS doctors who hardly get any exposure to such learning before entering post-graduation course.

Method: After obtaining consent, MBBS interns were oriented to the study and a pre-post questionnaire was administered to them to assess their knowledge and skill regarding NRP and was followed by NRP training. At the end of the posting and after completion of 6 months, the same questionnaire was administered for knowledge and skill assessment.

Results: $63.3 \%$ pre intervention could elicit correct response. $90.7 \%$ immediate post intervention could elicit correct response ( $p$-value <0.00001). 61.8\% after 6 month post intervention could elicit correct response ( $p$-value $<0.00001)$.

Conclusion: Decline in knowledge and skills is a significant barrier to the success of NRP training programs and possibly a hurdle in reducing new-born morbidity and mortality. Refresher training and structured practice has shown significant promise in success of Neonatal resuscitation programme.

Keywords: Neonatal Resuscitation Protocol, Birth asphyxia, Bag and Mask Ventilation, Chest compression.
\end{abstract}

\section{Introduction}

Globally, about one quarter of all neonatal deaths arecaused by birth asphyxia. Birth asphyxiais defined simply as the failure to initiate and sustain breathing at birth. Effective resuscitation at birth can prevent a large proportion of these deaths. ${ }^{[1]}$

Health care providers require training opportunities to acquire the knowledge and skills to appropriately manage neonates with critical illness and cardio-respiratory arrest. Competency in cardio-pulmonary resuscitation is a critical factor in determining successful outcomes in neonate. ${ }^{[2]}$

Practitioners who resuscitate infants at birth or at any time during the initial hospital admission should consider to follow these guidelines. Studies have demonstrated that use of simulation-based learning methodologies enhances performance in both simulated resuscitations and real-life clinical situations. $^{[3-4]}$ 
Retention of neonatal resuscitation skills vary from person to person. Retention of skill is more important in our setup for the doctors who pass out from medical college to enter practice as they hardly get any exposure to such learning before entering post-graduation course. ${ }^{[5-7]}$

\section{Materials and Methods}

Study Design - Longitudinal study.

Place of Study - Department of Paediatrics, HiTech Medical College and Hospital, BBSR.

Period of Study - November 2017 - October 2019.

\section{Inclusion criteria}

a. All the MBBS interns of batch 2012 2013 and 2013 - 2014 who were posted in neonatal ward. b. Those who gave consent for the study. Exclusion criteria - Those who were severely ill. Methodology

The MBBS interns were oriented to the study before recruiting them. Consent was obtained from each MBBS interns after they had thoroughly understood the research, a pre posting questionnaire was provided to MBBS interns to assess their knowledge and skill regarding NRP. It was followed by NRP hands on training with simulation exercise. At the end of the posting the same questionnaire was provided for knowledge and skill assessment. After 6 months, each of them was evaluated with the same questionnaire and then the skill and knowledge retention of NRP was assessed.

\section{Results}

\section{Knowledge Assessment}

Table 1(a). At level of pre-intervention and immediate post-intervention

\begin{tabular}{|c|c|c|c|c|c|}
\hline \multirow{2}{*}{ Response* } & \multicolumn{2}{|c|}{ Pre-Intervention } & \multicolumn{2}{|c|}{ Post-Intervention } & \multirow{2}{*}{ Chi-square } \\
\cline { 2 - 5 } & Frequency & Percent & Frequency & Percent & \multirow{2}{*}{27.0891} \\
\hline Correct & $\mathbf{1 7 8}$ & $\mathbf{8 5 . 2}$ & $\mathbf{2 0 3}$ & $\mathbf{9 9 . 1}$ & 0.9 \\
\hline Incorrect & 31 & 14.8 & 2 & 100.0 & $(p$-value is 0.00001$)$ \\
\hline Total & 209 & 100.0 & 205 &
\end{tabular}

Table 1(a) shows 209 interns were administered pre-intervention questionnaire out of which $85.2 \%$ could elicit correct response while 205 interns were administered immediate post-intervention questionnaire (following 4 numbers of loss of data) out of which $99.1 \%$ could elicit correct response.

The response was found to be statistically highly significant ( $p$-value $<0.00001)$. The chi-square statistic is 27.0891 . The $p$-value is 0.00001 . This result is significant at $p<0.05$.

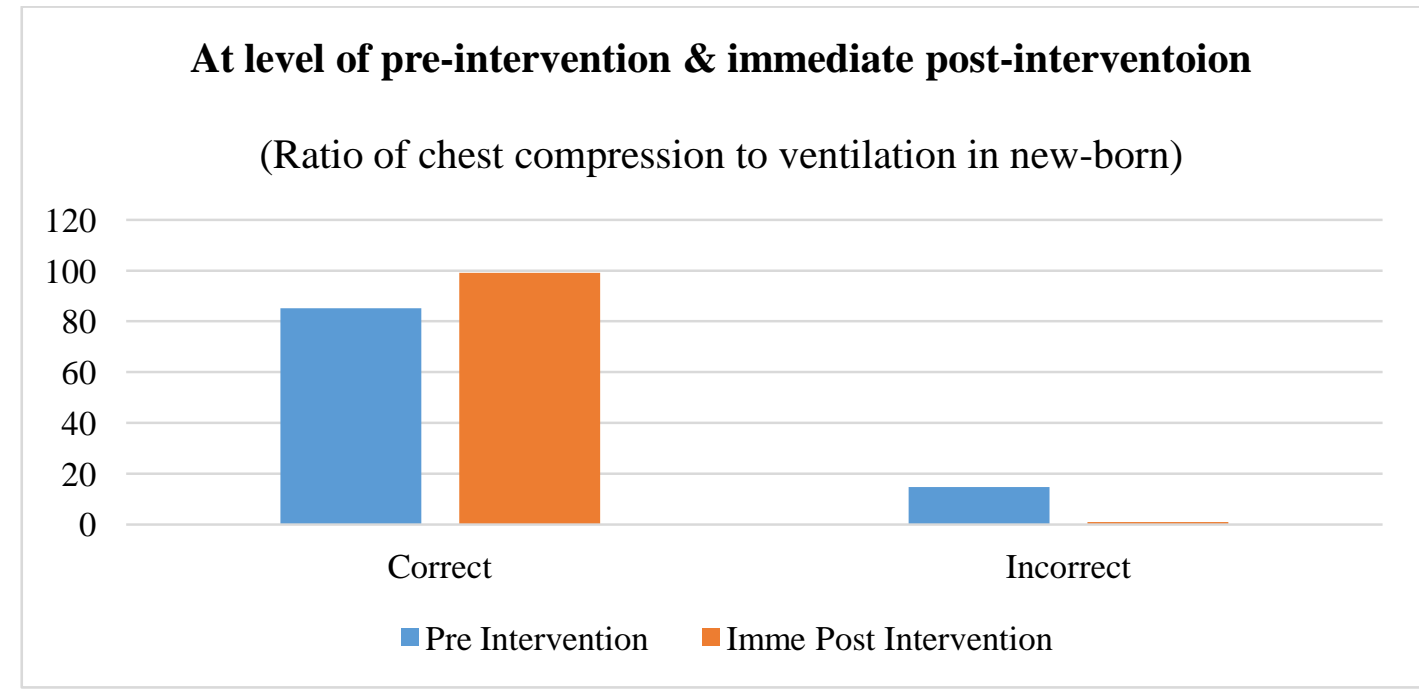

Diagram 1 
Table 1(b). At level of immediate post-intervention and 6 Months post-intervention

\begin{tabular}{|c|c|c|c|c|c|}
\hline \multirow{2}{*}{ Response* } & \multicolumn{2}{|c|}{ Post-Intervention } & \multicolumn{6}{|c|}{ 6 month Post-Intervention } & \\
\cline { 2 - 5 } & Frequency & Percent & Frequency & Percent & Chi-Square \\
\hline Correct & $\mathbf{2 0 3}$ & $\mathbf{9 9 . 1}$ & $\mathbf{1 2 6}$ & $\mathbf{6 1 . 8}$ & \multirow{2}{*}{ 90.2194 } \\
\hline Incorrect & 2 & 0.9 & 78 & 38.2 & ( $p$-value is 0.00001$)$ \\
\hline Total & 205 & 100.0 & 204 & 100.0 & \\
\hline
\end{tabular}

*(Ratio of chest compression to ventilation in new-born)

Table 1(b) shows 205 interns were administered The response was found to be statistically highly post intervention questionnaire out of which 99.1\% could elicit correct response while 204 interns were administered 6 months post significant ( $p$-value <0.00001). The chi-square statistic is 90.2194 . The $p$-value is 0.00001 . This result is significant at $p<0.05$. intervention questionnaire (following 1 numbers of loss of data) out of which $61.8 \%$ could elicit correct response.

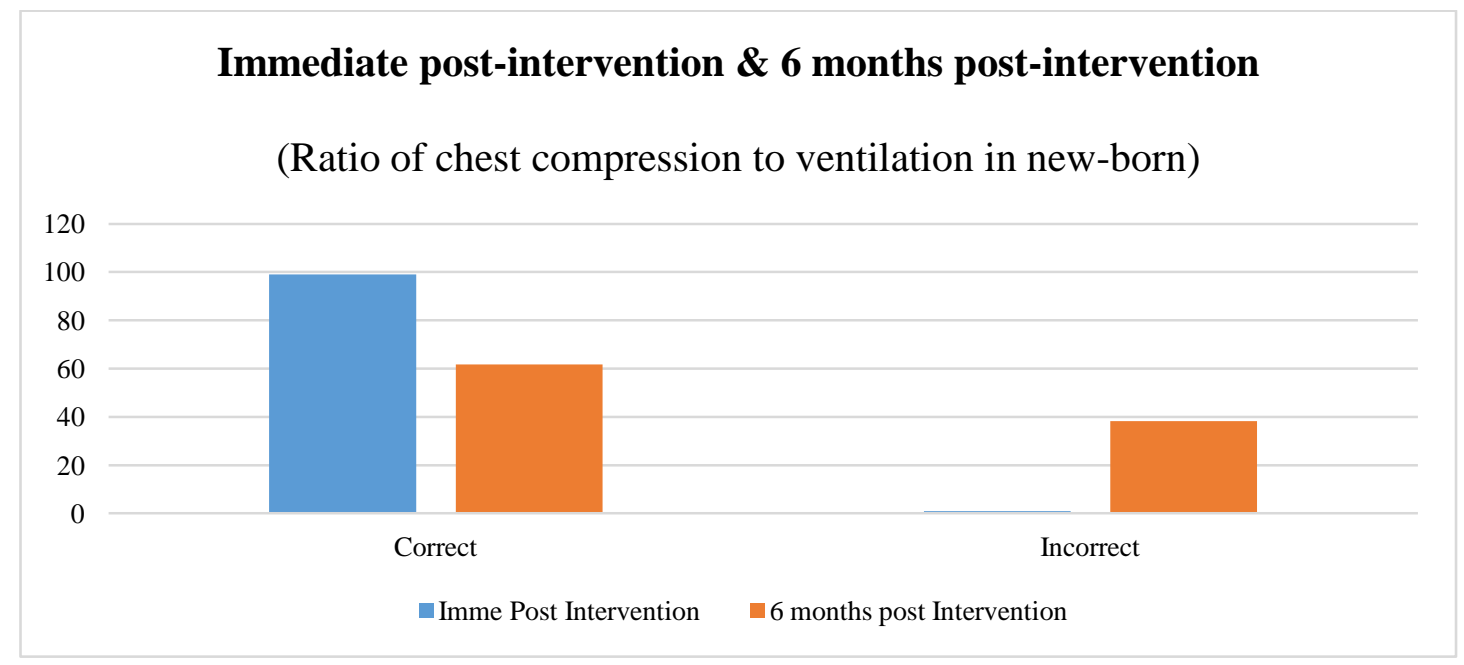

\section{Diagram 2}

Table 1(c). At level of pre-intervention and 6 months post-intervention

\begin{tabular}{|c|c|c|c|c|c|}
\hline \multirow{2}{*}{ Response* } & \multicolumn{2}{|c|}{ Pre Intervention } & \multicolumn{2}{|c|}{6 month post Intervention } & \\
\cline { 2 - 5 } & Frequency & Percent & Frequency & Percent & Chi-Square \\
\hline Correct & $\mathbf{1 7 8}$ & $\mathbf{8 5 . 2}$ & $\mathbf{1 2 6}$ & $\mathbf{6 1 . 8}$ & \multirow{2}{*}{29.1045} \\
\hline Incorrect & 31 & 14.8 & 78 & 38.2 & \multirow{2}{*}{$(p$-value is 0.00001$)$} \\
\hline Total & 209 & 100.0 & 204 & 100.0 & \\
\hline
\end{tabular}

*(Ratio of chest compression to ventilation in new-born)

Table 1(c) shows 209 interns were administered pre-intervention questionnaire out of which $85.2 \%$ could elicit correct response while 204 interns were administered 6 months post-intervention questionnaire (following 5 numbers of loss of The response was found to be statistically highly significant ( $p$-value <0.00001). The chi-square statistic is 29.1045 . The $p$-value is 0.00001 . This result is significant at $p<0.05$. data) out of which $61.8 \%$ could elicit correct response. 


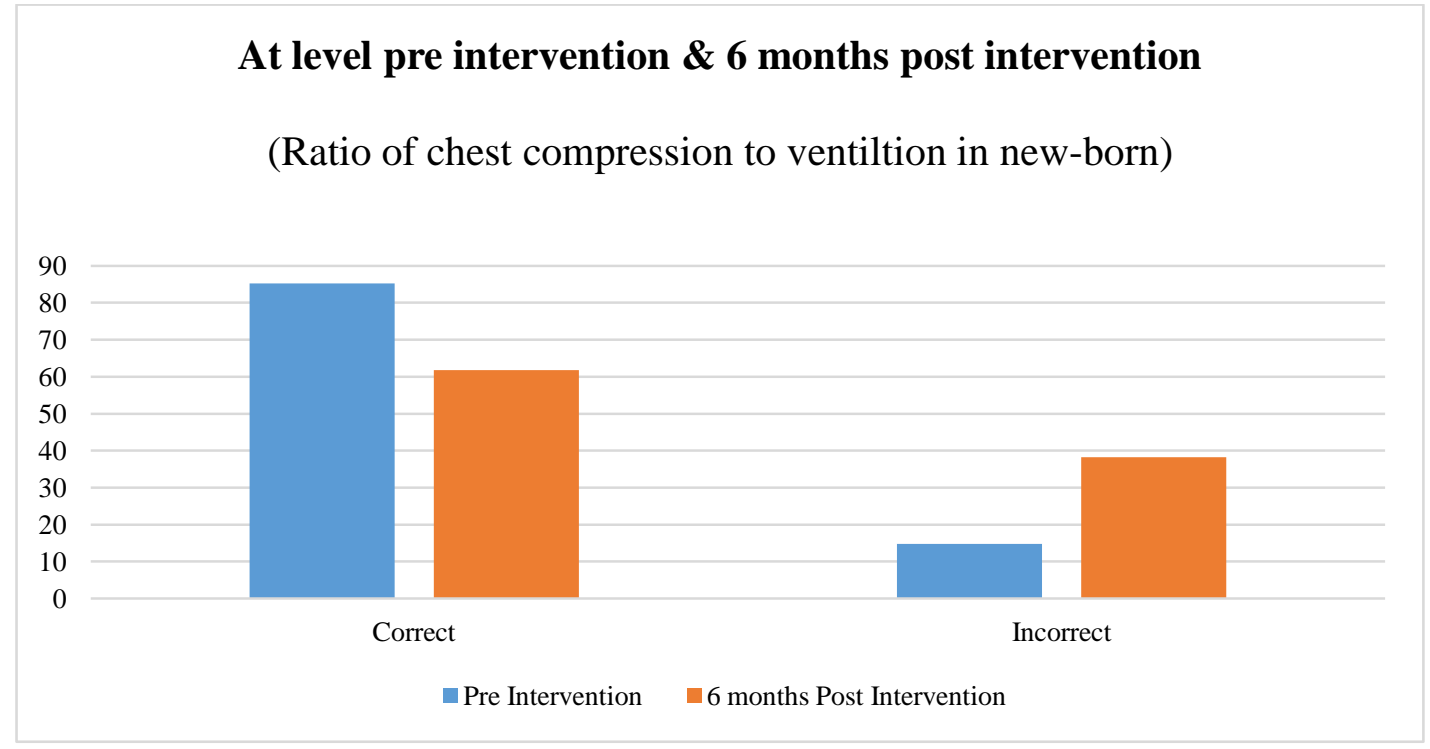

Diagram 3

\section{Skill Assessment}

Table 2. (a) At Level of Pre-Intervention and Immediate Post-Intervention

\begin{tabular}{|c|c|c|c|c|c|}
\hline \multirow[b]{2}{*}{ Response* $^{*}$} & \multicolumn{2}{|c|}{ Pre Intervention } & \multicolumn{2}{|c|}{ Post Intervention } & \\
\hline & Frequency & Percent & Frequency & Percent & Chi-Square \\
\hline Correct & 118 & 56.5 & 155 & 75.6 & \\
\hline Incorrect & 91 & 43.5 & 50 & 24.4 & 16.8996 \\
\hline Total & 209 & 100.0 & 205 & 100.0 & ( $p$-value is 0.000039$)$ \\
\hline
\end{tabular}

*(New-born is apnoeic and does not improve with initial steps, a positive pressure ventilation is started. First assessment of heart rate is 40 beats per minute. After 30 seconds of positive pressure ventilation that moves the chest, her heart rate is 80 beats per minute. Next steps in management)

Table 2(a) shows 209 interns were administered pre intervention questionnaire out of which $56.5 \%$ could elicit correct response while 205 interns were administered immediate post intervention questionnaire (following 4 numbers of loss of data) out of which $75.6 \%$ could elicit correct response.

The response was found to be statistically highly significant ( $p$-value <0.000039). The chi-square statistic is 16.8996 . The $p$-value is 0.000039 . This result is significant at $p<0.05$.

\section{At level of Pre Intervention \& Immediate post Inetrvention}

(New born is apnoeic \& does not improve with initial steps, a PPV is started. First assessment of heart are is 40 beats per minute. After 30 seconds of positive ventilation that moves the chest, $h$

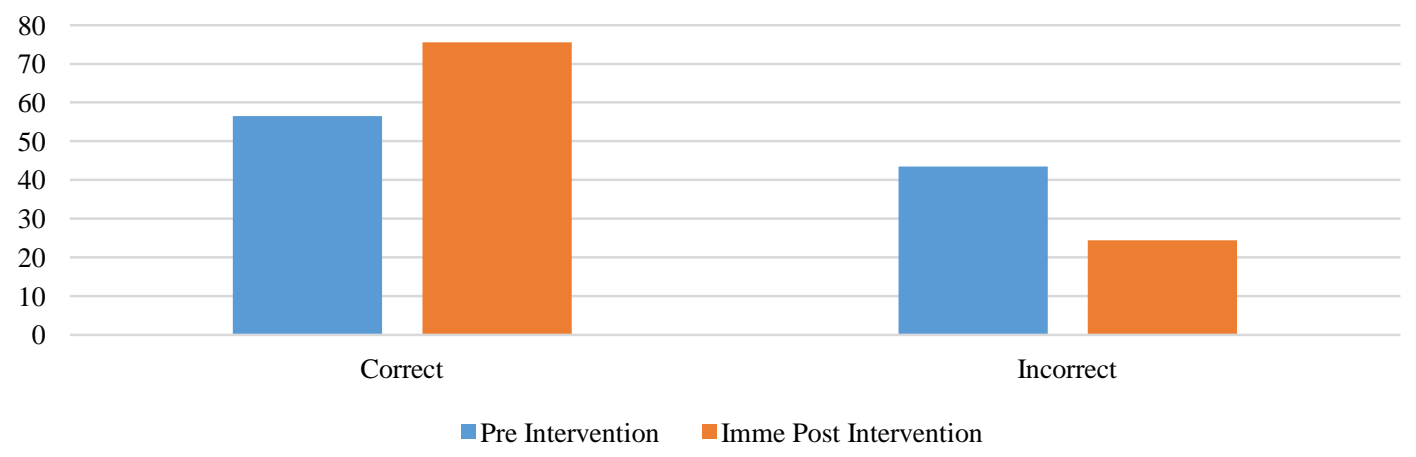

\section{Diagram 4}


Table 2. (b) At Level of Immediate Post-Intervention And 6 Months Post-Intervention

\begin{tabular}{|c|c|c|c|c|c|}
\hline \multirow{2}{*}{ Response* } & \multicolumn{2}{|c|}{ Post-Intervention } & \multicolumn{2}{|c|}{6 month Post-Intervention } & \\
\cline { 2 - 5 } & Frequency & Percent & Frequency & Percent & Chi-Square \\
\hline Correct & $\mathbf{1 5 5}$ & $\mathbf{7 5 . 6}$ & $\mathbf{1 3 3}$ & $\mathbf{6 5 . 2}$ & \multirow{2}{*}{5.3228} \\
\hline Incorrect & 50 & 24.4 & 71 & 34.8 & ( $p$-value $<0.021049)$ \\
\hline Total & 205 & 100.0 & 204 & 100.0 & \\
\hline
\end{tabular}

*(New born is apnoeic and does not improve with initial steps, a PPV is started. First assessment of heart are is 40 beats per minute. After 30 seconds of positive pressure ventilation that moves the chest, her heart rate is 80 beats minutes. Next steps in management).

Table 2(b) shows 205 interns were administered immediate post-intervention questionnaire out of which $75.6 \%$ could elicit correct response while 204 interns were administered 6 months postintervention questionnaire (following 1 numbers of loss of data) out of which $65.2 \%$ could elicit correct response.

\section{At level of Post Intervention \& 6 months post Inetrvention}

(New born is apnoeic \& does not improve with initial steps, a PPV is started. First assessment of heart are is 40 beats per minute. After 30 seconds of positive ventilation that moves the chest, $h$

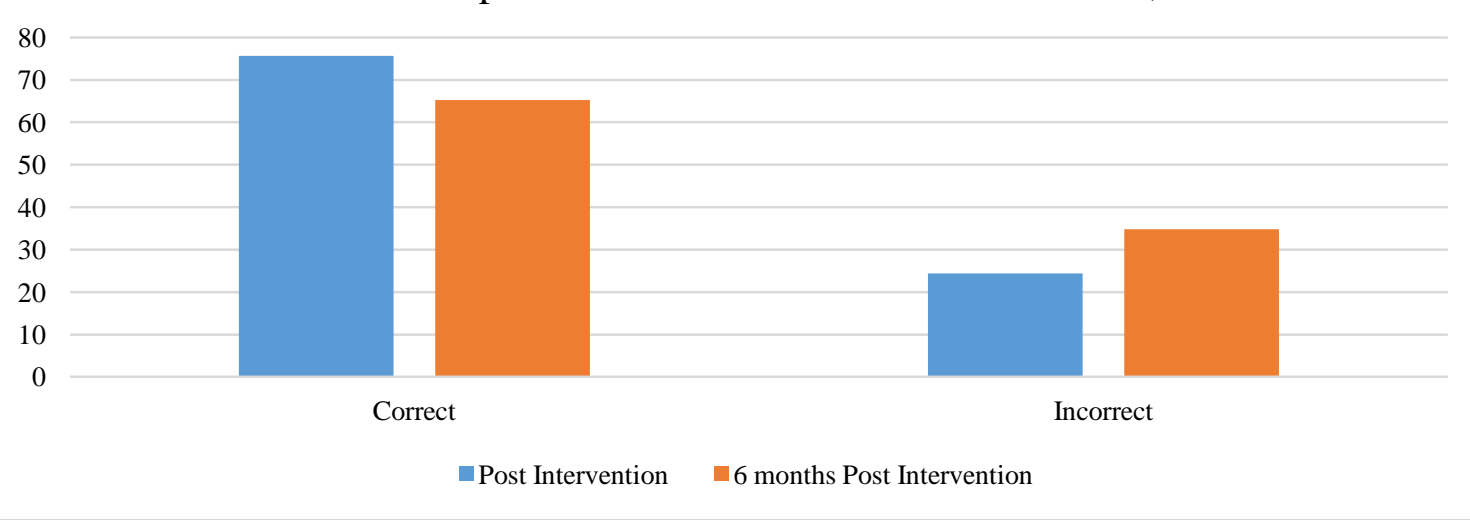

\section{Diagram 5}

Table 3. (c) At Level of Pre-Intervention And 6 Months Post-Intervention

\begin{tabular}{|c|c|c|c|c|c|}
\hline \multirow{2}{*}{ Response* } & \multicolumn{2}{|c|}{ Pre-Intervention } & \multicolumn{2}{|c|}{6 month Post-Intervention } & \multirow[b]{2}{*}{ Chi-Square } \\
\hline & Frequency & Percent & Frequency & Percent & \\
\hline Correct & 118 & 56.5 & 133 & 65.2 & \multirow{3}{*}{$\begin{array}{c}3.3055 \\
(p \text {-value }<0.069048)\end{array}$} \\
\hline Incorrect & 91 & 43.5 & 71 & 34.8 & \\
\hline Total & 209 & 100.0 & 204 & 100.0 & \\
\hline
\end{tabular}

*(New born is apnoeic and does not improve with initial steps, a PPV is started. First assessment of heart are is 40 beats per minute. After 30 seconds of positive pressure ventilation that moves the chest, her heart rate is 80 beats minutes. Next steps in management)

Table 3(c) shows 209 interns were administered pre-intervention questionnaire out of which $56.5 \%$ could elicit correct response while 204 interns were administered 6 months post-intervention questionnaire (following 5 numbers of loss of data) out of which $65.2 \%$ could elicit correct response. 


\section{At level of Pre-Intervention \& 6 months Post-Inetrvention}

(New born is apnoeic \& does not improve with initial steps, a PPV is started. First assessment of heart are is 40 beats per minute. After

30 seconds of positive ventilation that moves the chest, he

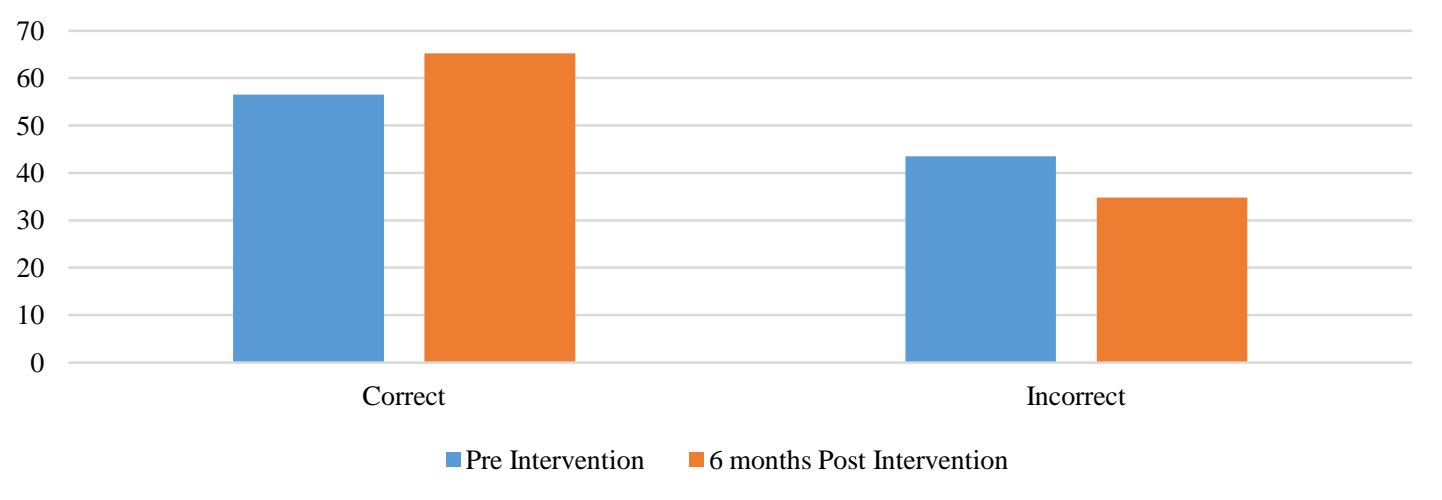

Diagram 6

\section{Discussion}

In this study, we found that pre-intervention knowledge among MBBS interns at our centre was adequate about evaluation of new-borns but their skills were low for conducting a proper neonatal resuscitation code in neonatal ward (diagram 1, 4). After the teaching and hands-on training, we found the knowledge and skills of the MBBS interns have significantly improved (diagram 1,4). We found similar results in study conducted by (Boo NY, Pong KM. Neonatal resuscitation training programme in Malaysia). ${ }^{\left[{ }^{8]}\right.}$ We conducted a post-intervention survey after 6 months of the study which revealed that the knowledge and skills of MBBS interns had significantly decreased from that of immediate post-intervention (diagram 2,5 ) but was slightly higher than that of pre-intervention (diagram 2,5).

On comparison of pre-intervention and 6 months post-intervention we found that knowledge was slightly higher in 6 months post-intervention than that of pre-intervention (diagram 3) but the level of skills were low and was same as that of preintervention state (diagram 6).

Structured teaching syllabus of Neonatal Resuscitation Protocol involving simulation labs are lacking in our medical curriculum. After completion of graduation course, it becomes difficult for training in Neonatal Resuscitation skills. Busy residency schedules and lack of resources act as barriers ${ }^{[9]}$. Doctors still are expected to learn resuscitation skills in the clinical setting, where there is little opportunity to correct poor techniques ${ }^{[10]}$. Given this situation, many junior doctors are not competent in carrying out effective Neonatal Resuscitation and may be a reason why good Neonatal Resuscitation skills are failing to develop, since the acquired knowledge cannot be put into practice.

Frequent Neonatal Resuscitation training needs to be organised for better retention of the knowledge and skills acquired and better performances during Neonatal Resuscitation ${ }^{[11]}$.

\section{Conclusion}

The introduction of routine and periodic NRP training programmes improved the NRP knowledge and skills among doctors, which is essential for improved survival of new born infants. A focus on acquisition of skills and preventing decline in knowledge and skills through regular practice will likely increase the impact of the training programs on new-born. ${ }^{[12]}$

\section{References}

1. Guideline on Basic New-born Resuscitation, 2012: $\operatorname{Pg} 2$. 
2. Cheng A, Brown L, Duff J, et al. Improving Cardiopulmonary Resuscitation with a CPR Feedback Device And Refresher Simulations (CPRCARES Study) : a multicentre, randomized trial. JAMA Pediatr. 2015; 169 (2): 137-44.

3. Montgomery C, Kardong-Edgren SE , Oermann MH, et al. Students satisfaction \&self-report of CPR Competency : Heart code BLS courses, Instructor led CPR courses \& monthly voice advisory manikin practice for CPR skill maintainance. Int J Nurs Educ Scholarsh. 2012;Pg. 1515-48.

4. Schwid HA, Rooke GA, Michalowski P, Ross BK.Screen-based anaesthesia simulation with debriefing improves performance in a mannequin-based anaesthesia simulator. Teach Learn Med. 2001;13: 92-96.

5. D. Trevisanuto, $P$. Ferrarese, $P$. Cavicchioli, et al. "Knowledge gained by Paediatric residents after neonatal resuscitation program courses," Paediatric Anaesthesia, 2005 , vol. 15, no. 11, pp. 944-47.

6. J. Singh, S. Santosh, J. P. Wyllie, et al., "Effects of a course in neonatal resuscitation - evaluation of an educational intervention on the standard of neonatal resuscitation," Resuscitation, 2006, vol. 68, no. 3, Pg. 385-89.

7. T. Xu, H.-S. Wang, H.-M. Ye et al., "Impact of a nationwide training program for neonatal resuscitation in China," Chinese Medical Journal, 2012, vol. 125, no. 8, Pg. 1448-56.

8. Boo NY, Pong KM. Neonatal resuscitation training programme in Malaysia): results of the first 2 years. J Paediatric Child Health 2001; 37: 118124).

9. Kambarami RA, Chirenje M, Rusakaniko S. Situational analysis of obstetric care services in a rural district in Zimbabwe. Cent Afr J Med 2000; 46: 154-157.

10. Couper ID, Thurley TD, Hugo JF. The neonatal resuscitation training project in rural South Africa. Rural Remote Health 2005; 5: 459

11. Halamek LP, Kaegi DM, Gaba DM, et al. Time for a new paradigm in paediatric medical education: teaching neonatal resuscitation in a simulated delivery room environment. Paediatrics 2000; 106: e45.

12. Mduma E, Ersdal H, Svensen E, Kidanto H, Auestad B, Perlman J. Frequent brief on-site simulation training and reduction in 24-h neonatal mortality- an educational intervention study. Resuscitation. 2015; 93:1-7). 